\title{
CIENCIA\&SALUD
}

\section{OSTOMÍAS DE ALTO GASTO EN PACIENTES ADULTOS}

\section{HIGH - OUTPUT STOMA IN ADULT PATIENTS}

Sofía Segreda Castro ${ }^{1}$ Cristine Segura Araya ${ }^{2}$

1 Médico general y nutricionista. Trabajador independiente, San José Costa Rica

1 Médico general.Trabajador independiente, San José Costa Rica

Contacto: sofi_segreda@hotmail.com cristhine_22@hotmail.com

\section{RESUMEN}

La ostomía de alto débito es una de las complicaciones que pueden presentar los pacientes portadores de patologías que requieran la construcción de un estoma. A través de los años, se ha visto un incremento en la necesidad de establecer no solo una definición clara sobre este concepto, sino también un consenso en cuanto a su manejo.

Portar un estoma puede ser una condición temporal o permanente. Esta situación aumenta el riesgo de consecuencias negativas, ya sea a corto o a largo plazo y puede significar un aumento en los costos de servicios de salud al ser una patología de difícil manejo y que requiere constante monitoreo.

Palabras clave: Enfermedad celiaca, gluten, absorción intestinal, desnutrición.

\section{Cómo citar:}

Segreda Castro, S., \& Segura Araya, C. (2021). Ostomías de alto gasto en pacientes adultos. Revista Ciencia Y Salud, 5(1), Pág. 102 - 110.

\section{ABSTRACT}

High - output stoma is one of the complications that can be present in patients with pathologies that require a stoma. Over the years, there has been an increase in the need to establish not only a clear definition of this concept, but also a consensus regarding its management.

Carrying a stoma can be a temporary or a permanent condition. This increases the risk of negative consequences, either in the short or long term, since it can mean an increase in the costs of health services as it is a pathology that is difficult to manage and requires constant monitoring.

Keywords: High output stoma, nutrition, complications, psychology, treatment.

Recibido: 22/Oct/2020

Aceptado: $10 /$ ene/2021

Publicado: $15 / \mathrm{feb} / 2021$

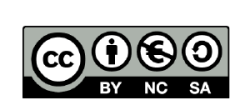




\section{CIENCIA\&SALUD}

\section{INTRODUCCIÓN:}

El término ostomía intestinal se refiere al acto quirúrgico en donde se exterioriza una porción de intestino hacia la pared abdominal con el fin de crear una nueva manera de eliminar desechos del cuerpo o una vía de alimentación como consecuencia de alguna condición médica que padezca el paciente (1) (2). De hecho, es un procedimiento no tan infrecuentemente realizado. En países como Estados Unidos se efectúan más de 100000 de estas cirugías al año (3).

La formación de un estoma está indicado en diferentes patologías como por ejemplo la enfermedad de Crohn refractaria al tratamiento farmacológico, enfermedad vascular y neoplasias. Muchas de las cirugías citadas anteriormente involucran la formación de una ostomía para aliviar síntomas, proteger una anastomosis con alto riesgo de fuga y manejar la incontinencia secundaria (4).

Aunque se sabe que los portadores de ostomías tienen una limitación mínima en sus actividades de vida diaria (4), se pueden presentar diversas dificultades, estimándose una incidencia de $21-70 \%$ (3). Algunas de las complicaciones citadas en la literatura son várices periostomales, hernia paraestomal, ostomía de alto gasto, prolapso, alteraciones dermatológicas, infecciones, estenosis, retracción, entre otras (4).

Durante los primeros días posteriores a la formación del estoma, en condiciones normales, el flujo de heces se ve aumentado para posteriormente sufrir una adaptación intestinal y en consecuencia disminuir rápidamente la expulsión de materia fecal. Cuando esta adaptación es prolongada o falla, los pacientes pierden grandes cantidades de fluidos, presentando así lo que se conoce como ostomía de alto débito, en la cual se pierden grandes cantidades de sodio, magnesio y agua; y además pueden presentarse otras complicaciones como pérdida de peso por malabsorción y subnutrición (4) (5). De hecho, la ostomía de alto gasto se ha identificado como precursor de deshidratación y disfunción renal, con una incidencia de aproximadamente $1-17 \%$ y un porcentaje de readmisión hospitalaria de 4-43\% (4). A su vez, se ha registrado que en las personas portadoras de ileostomías, aproximadamente el 16\% desarrollará de forma temprana un alto gasto y el $7 \%$ de estos individuos podría presentar esta condición de forma prolongada, requiriendo un manejo continuo (6). Por todo lo anteriormente explicado, se considera un tema de suma importancia para ser estudiado e investigado.

\section{DEFINICIÓN:}

Ostomía se refiere al término utilizado para el procedimiento quirúrgico en el cual se realiza la apertura (estoma) de una o dos vísceras huecas, por lo tanto existe una gran variedad de ostomías, como por ejemplo: ileostomías, traqueostomías, ureterostomías, colostomías, entre otras. Si bien existen diversas razones para la confección de una colostomía o una ileostomía, algunas de sus indicaciones son: cirugía de urgencia para descomprimir una obstrucción distal, falla o resección del complejo esfinteriano anal, resección colorrectal en donde se realiza una anastomosis pero esta debe ser protegida, reducción en el paso de contenido fecal a través de un segmento de intestino que se encuentre comprometido por una patología.

Una ostomía con gasto adecuado debe tener un débito entre $600-800 \mathrm{ml}$ diarios a través de una ileostomía. El límite a partir del cual se considera un alto gasto no está descrito de manera consensuada, pero se cree que una ileostomía de alto débito se define como el déficit mayor a $2000 \mathrm{ml}$ en 24 horas, siendo contabilizado desde el post operatorio inmediato. Además, se pueden dividir según el tiempo de evolución en tempranas y tardías, encontrándose las primeras en una fase postoperatoria menor a tres semanas y las últimas en un período posterior a la cirugía mayor a tres semanas (4) (6) (7) (8) (17) (21).

\section{CAUSAS DEL ALTO GASTO:}

Distintos factores de riesgo podrían contribuir a la formación de un estoma de elevado débito, pero no se ha identificado una causa específica para su desarrollo. Algunos de los contribuyentes a esta condición son 


\section{CIENCIA\&SALUD}

el síndrome de intestino corto, fármacos, infecciones gastrointestinales, sepsis intraabdominal (4), obstrucción intestinal, íleo paralítico y absceso intraabdominal.

Otro elemento influyente en esta condición es la diabetes, la cual además de asociarse con complicaciones postoperatorias, también es un potencial factor para el desarrollo de una ostomía de alto débito, ya que disminuye la función motora y crea una proliferación bacteriana anormal, principalmente en intestino delgado, causando producción de gas y por ende elevación de la presión intestinal (9).

Por otro lado, en cuanto a los mecanismos influyentes de esta patología, se encuentra la deficiencia de ácido biliar, que inhibe la absorción lipídica y conlleva a cambios en la flora intestinal, teniendo como consecuencia el aumento en las excretas (9).

Por último, es de suma importancia recalcar que los pacientes con ostomías presentan cambios fisiológicos en su cuerpo. Estos cambios están en relación con la disminución en la absorción de agua, electrolitos y con la falta de continencia a la hora de expulsar las heces (21).

\section{CITA PREQUIRÚRGICA:}

Con el fin de reducir las compllicaciones posteriores a la cirugía, se recomienda programar una cita prequirúrgica para marcar el sitio del estoma con el paciente en las posiciones supina, de pie y sentado. El estoma se posicionará sobre el recto abdominal en una superficie plana de $5 \mathrm{~cm}$ y se deben tomar en consideración diversos factores como el hábito corporal del individuo, la agudeza visual y destreza del cirujano, el tipo de ostomía (en asa vs terminal, colostomía vs ilestomía) y el contorno abdominal (evitar sitios quirúrgicos previos, pliegues cutáneos, la cicatriz umbilical, la zona en donde se coloca el cinturón).

Además, en esta cita es importante hablar con el paciente y sus cuidadores sobre las implicaciones a las que conlleva un estoma. Algunas de estas complicaciones se mencionan a continuación (24).

\section{COMPLICACIONES:}

Como se mencionó anteriormente, algunas de las complicaciones en las personas ostomizadas son el prolapso, hernia paraestomal, várices periostomales, alteraciones dermatológicas, estenosis, retracción e infecciones (4). No obstante, en los portadores de ostomías de alto flujo existen algunas dificultades muy importantes para tomar en consideración:

A. Alteraciones dermatológicas periostomales: ocurren en el $43 \%$ de los pacientes ostomizados, siendo frecuentes en los portadores de ileostomías y de ostomías de alto gasto (3) (8). Además, se debe tomar en cuenta que con el fin de prevenir afecciones dermatológicas, el cirujano debe crear una colostomía de más de $1 \mathrm{~cm}$ o una ileostomía de aproximadamente $2-3 \mathrm{~cm}$ para así reducir el contacto directo entre los desechos y la piel (3).

En caso de que las personas presenten estas complicaciones, se puede utilizar terapia tópica para promover la cicatrización y proteger la piel, aplicar utensilios convexos que mejoren la protrusión del estoma y el anillo de barrera cutánea para mejorar el sistema de sellado y siempre tener en cuenta que el alto gasto puede dificultar el acople de las bolsas recolectoras. Si a pesar de todo lo anterior la afección persiste, deberá considerarse la exploración quirúrgica (3).

En este mismo grupo se encuentra la separación mucocutánea, que ocurre en el $28 \%$ de individuos en el post operatorio inmediato y se refiere a la disociación de la ostomía de la piel perisotomal circundante. Generalmente se produce como consecuencia de una técnica inadecuada en donde se aplica tensión excesiva, pero también puede desencadenarse por diabetes, infecciones, abuso en la utilización del cauterio en mucosa o piel y factores que alteren la cicatrización (3). 


\section{CIENCIA\&SALUD}

La detección temprana y el manejo agresivo de la herida son vitales en el tratamiento de la separación mucocutánea. Cuando este defecto es pequeño, puede manejarse con materiales de relleno absortivos como el talco, pero en defectos grandes o dehiscencia circunferencial se necesita la revisión quirúrgica para evitar complicaciones a largo plazo como la estenosis o retracción (3).

B. Infección por Clostridium difficile: algunos factores de riesgo para padecer esta infección son múltiples comorbilidades, malnutrición, exposición hospitalaria, inmunosupresión, uso de inhibidores de bomba de protones, edad y pacientes que se someten a cirugía electiva. Las personas colectomizadas presentan un riesgo único de contraer esta afección por la disrupción física en la microflora colónica, reportándose cada vez mayor cantidad de casos de infecciones por este microorganismo y como consecuencia generándose un impacto negativo en los resultados post quirúrgicos de estas personas (10).

C. Desbalance de fluidos y electrolitos: se considera la causa más frecuente de readmisión hospitalaria en el post operatorio temprano. Esta complicación es un factor de riesgo conocido para lesión renal aguda y enfermedad renal crónica. Algunos estudios sugieren que los individuos portadores de ileostomías presentan mayor probabilidad de deshidratación clínica en los primeros tres a ocho días posteriores a la cirugía, por lo que se debe tener extrema precaución en cuanto al balance hidroelectrolítico en este periodo de tiempo (3).

D. Malabsorción de sales biliares: la resección ileal mayor a $100 \mathrm{~cm}$ produce una grave malabsorción de sales biliares que podría causar una reducción progresiva en la reserva de ácidos biliares, insuficiente solubilización de vitaminas liposolubles y grasas, produciendo un aumento en las excretas intestinales (17).

E. Déficit de vitamina B12: la carencia de esta vitamina depende de la longitud de íleon resecado en cada persona, ya que su absorción se limita únicamente a esta porción de intestino. La extracción ileal mayor a $60 \mathrm{~cm}$ conllevará a una depleción de vitamina B12 en el organismo, pudiendo causar anemia megaloblástica o perniciosa (17).

\section{MANEJO:}

El manejo de las ostomías de alto gasto suele ser bastante retador tanto a nivel farmacológico como a nivel nutricional y su combinación con deshidratación y/o malnutrición es bastante frecuente, sobre todo en pacientes portadores de ileostomías. Entre los factores más importantes a tomar en cuenta para decidir el rumbo del tratamiento son la funcionalidad y la longitud del intestino remanente (8).

Es importante recalcar que más del 50\% de los pacientes con estomas de alto débito necesitarán tratamiento médico prolongado y que hasta el $37 \%$ de estas personas podrían necesitar en algún momento nutrición parenteral y administración de fluidos (8). No obstante, previo al inicio del manejo nutricional y farmacológico, debe identificarse y tratarse la causa desencadenante del excesivo gasto (4).

A. Tratamiento farmacológico: Los medicamentos mencionados a continuación son utilizados en estos individuos con el fin de reducir el gasto a través de sus diferentes mecanismos de acción, deben ser administrados anterior a la ingesta de cualquier tipo de alimento para que puedan cumplir su función (11).

1. Inhibidores de bomba de protones: se unen de forma covalente a la hidrógeno potasio ATPasa por lo que ocasiona la inhibición irreversible en la excreción de ácido en el tracto gastrointestinal (12).

En un estudio realizado por De Vries, et al., se utilizó omeprazol vía oral o intravenosa a dosis de 40 mg una o dos veces al día, con el fin de disminuir el alto débito y este mostró ser más eficaz que el placebo o la ranitidina (12). 


\section{CIENCIA\&SALUD}

2. Fármacos antimotilidad: son agonistas de receptores opioides que disminuyen la actividad del plexo mientérico y por ende enlentecen el tracto gastrointestinal (12). Estos agentes controlan el tiempo del tránsito intestinal y así maximizan la absorción de nutrientes (5).

De Vries, et al., utilizaron loperamida vía oral a dosis que varían desde 6 mg al día hasta 16 mg por día dividido en cuatro tomas (dosis máxima), con el objetivo de conseguir una reducción media de $22-45 \%$ en el gasto de la ostomía. También se ha estudiado el uso de codeína a dosis que van desde los $10 \mathrm{mg}$ tres veces al día hasta máximo 20 mg tres veces al día vía oral, pero este medicamento presentó mayor tasa de efectos secundarios (12) (18).

3. Agentes que aumentan la respuesta adaptativa: teduglutida es un medicamento análogo del péptido 2 similar al glucagón (GLP-2) que actúa aumentando el flujo sanguíneo mesentérico, promoviendo el crecimiento de mucosa, disminuyendo la excreción de ácido y la motilidad gástrica. Este fármaco se administra por vía subcutánea y ha sido aprobado en falla intestinal crónica debida a síndrome de intestino corto. Se cree que esta droga podría disminuir el alto débito, sin embargo, al ser sumamente costosa debe reservarse únicamente para individuos refractarios al manejo farmacológico que se encuentren gravemente enfermos (13).

4. Antihistamínicos antagonistas del receptor a 2: estos medicamentos actúan mediante el bloqueo de la acción de la histamina en los receptores h2 de las células parietales en el estómago, lo que resulta en una baja de la secreción ácida (12).

Se sabe que el tratamiento con antihistamínicos como la ranitidina y cimetidina reducen la secreción gastrointestinal. Sin embargo, se prefiere la utilización de inhibidores de bomba de protones para también mitigar otros síntomas como reflujo gastroesofágico o tratar úlceras pépticas.

A pesar de lo descrito anteriormente, siempre se debe tener en cuenta que los medicamentos pueden causar efectos secundarios. Algunos de los causados por los inhibidores de bomba de protones son la hipomagnesemia y mayor riesgo de fracturas (12) (19) y entre los efectos causados por los antihistamínicos antagonistas del receptor $\alpha 2$ se encuentran la taquifilaxia con el uso intravenoso desde las primeras 42 horas de administración, somnolencia, letargo y confusión (20).

En cuanto a las dosis de estos agentes, se ha comprobado que la utilización de ranitidina 150 mg al día vía intravenosa o de cimetidina $200-800 \mathrm{mg}$ al día vía intravenosa disminuye significativamente la producción estomal (12).

5. Electrolitos: El paciente debe aprender a reconocer los síntomas de deshidratación, entre los cuales se encuentran debilidad, boca seca, espasmos musculares, reducción en la micción, sed, mareos y además monitorizar el gasto a través del estoma. En esta misma línea, el individuo debe registrar todas las ingestas, excretas y vigilar su peso; mientras que el personal en salud debe realizar análisis de sodio urinario, perfil electrolítico que incluya magnesio y marcadores nutricionales (17).

6. Colestirarmina: Secuestra ácidos biliares no absorbidos y disminuye la absorción de cationes divalentes. Se debe tener en cuenta que su uso prolongado, especialmente posterior a la resolución de la patología desencadenante del alto gasto, puede llevar a malabsorción de cationes divalentes y vitaminas liposolubles. La dosis recomendada de colestiramina es de 2 - $4 \mathrm{~g}$ dos veces al día (22) (4).

7. Octreótido: Aumenta la absorción de sodio y agua, disminuye el tránsito intestinal, sin embargo, reduce la síntesis de proteínas dificultando la adaptación intestinal y eleva el riesgo de cálculos biliares, por lo tanto únicamente se recomienda su uso si luego de dos semanas con los tratamientos mencionados anteriormente, el alto gasto continúa (22).

Por último, en cuanto al manejo de fluidos y electrolitos, en el año 2015 Arenas, J., et al., desarrollaron este tema en el protocolo para la detección y manejo nutricional en estomas de alto gasto, en donde se propone 


\section{CIENCIA\&SALUD}

lo siguiente (4):

1. Detección y tratamiento de la causa desencadenante.

2. Etapa I o tratamiento inicial: se debe disminuir la pérdida de electrolitos y restringir la ingesta de fluidos a 500-1000 ml/día, siendo las bebidas isotónicas la mejor opción y teniendo que evitar los líquidos hipotónicos como: alcohol, té y café. Además, debe valorarse hidratación intravenosa, loperamida previo a las comidas y dependiendo de los resultados en los laboratorios podría requerirse suplementación vía oral o intravenosa de electrolitos y vitamina B12. Posterior a lo citado anteriormente, se determina el gasto estomal durante 48-72 horas, si el alto gasto se resolvió en este lapso de tiempo, se puede aumentar la ingesta oral de líquidos y modular los medicamentos.

3. Etapa II: si permanece el alto débito a través de la ostomía se realiza tratamiento de seguimiento. En esta etapa se continúa con la misma restricción hídrica mencionada y se inicia ingesta de sales de rehidratación oral de 500-1000 ml/día como la única fuente de fluidos vía oral. También se aumenta la dosis de loperamida hasta máximo $16 \mathrm{mg} /$ día, se inicia con omeprazol o se aumenta la dosis si se encontraba prescrito anteriormente y si el enfermo presenta malabsorción de grasas podría considerarse el uso de colestiramina $4 \mathrm{~g}$ dos veces al día previo al desayuno y cena. Es muy importante continuar con el monitoreo del paciente y brindar suplementación electrolítica en caso de ser necesario. Si luego de 48-72 h el paciente continúa con un elevado gasto a través de la ostomía se continuará con la siguiente etapa.

4. Etapa III: al llegar a este estadio se continúa con el monitoreo de ingesta de fluidos, se indica suplementación de vitaminas lipo e hidrosolubles, se mantiene la loperamida y se agrega codeína antes de las comidas si no existe contraindicación. Si la malabsorción de grasas persiste, se recomienda incrementar la dosis de colestiramina. Si luego de dos semanas el gasto es mayor a $2000 \mathrm{ml}$, se puede agregar octreótido, pero si en 3-5 días no ha sido efectivo se suspenderá el tratamiento con este agente.

B. Nutrición: el objetivo de la terapia nutricional es mejorar la consistencia y disminuir el volumen de materia fecal. Los planes dietéticos deben ser individualizados y basados en las necesidades de cada paciente, manteniendo una adecuada hidratación y considerando la posible necesidad de nutrición parenteral (5). A pesar de ello, los profesionales encargados del manejo de estas personas, debe tomar en cuenta que la nutrición parenteral se asocia con mayores efectos metabólicos, complicaciones y estancias hospitalarias prolongadas, por lo que se reserva únicamente para pacientes que no logren llenar los requerimientos por vía enteral (14).

Por otro lado, los pacientes con 60-100 cm de yeyuno intactos podrían presentar grandes gastos estomales y absorber menos del 35\% de la energía disponible, requiriendo así la nutrición parenteral (14).

Además, las guías ASPEN recomiendan un aporte proteico de 1.5-2.0 g/Kg/día y energético de 35-60 Kcal/ $\mathrm{kg} /$ día para síndrome de intestino corto con yeyunostomía terminal. Aunado a esto, debe tomarse en cuenta tanto la ingesta del paciente como el volumen perdido a través del estoma para realizar el cálculo de todos los requerimientos nutricionales (5).

En esta misma línea, se recomienda que el paciente realice pequeñas ingestas de alimentos cada 3-4 horas, evite el consumo de líquidos durante las comidas, aumente temporalmente el contenido de sal en los alimentos para así promover la reabsorción de líquidos y que prolongue la inclusión dietética de fibras y vegetales a más de 6-8 semanas posterior a la construcción estomal, ya que en estas personas la adaptación intestinal se encuentra retardada. Sin embargo, se conoce muy poco sobre el uso de fibra soluble en esta población, pero se sabe que la fibra insoluble se encuentra contraindicada en ellos por el riesgo de obstrucción intestinal (4).

Por útlimo, el Glutapak, es un suplemento utilizado en nutrición parenteral en el que cada $15 \mathrm{~g}$ de polvo contiene $10 \mathrm{~g}$ de L-Glutamina, $5 \mathrm{~g}$ de maltodextrina y 100 millones de unidades formadoras de colonias de Lactobacillus reuteri y se encuentra indicado para la protección y restauración de la salud intestinal, 


\section{CIENCIA\&SALUD}

síndrome de intestino corto, malabsorción, diarrea, intolerancia gastrointestinal, pacientes con nutrición parenteral, entre otros (23).

\section{PSICOLOGÍA EN PACIENTES OSTOMIZADOS:}

Como se ha mencionado, el componente psicológico es esencial para lograr una adecuada recuperación en estos individuos. Al experimentar algún tipo de cambio corporal, la persona se verá afectada física y mentalmente, por lo que es importante llevar un seguimiento psicológico individualizado con cada uno de ellos (15).

El portar un equipo colector se asocia a sentimientos negativos, que incluyen desde la vergüenza, tristeza, miedo y desamparo, menor optimismo, esperanza y relaciones familiares en las que hay tensión por la nueva condición del paciente. Todos estos sentimientos pueden originar vivencias de tipo auto despreciativas, deterioro de la autoimagen y trastornos de identidad que pueden ligar sentimientos de pérdida de salud y de autoestima, incluso de mutilación. Por lo anteriormente explicado, los individuos que presentan esta condición se ven realmente forzados a moldear su estilo de vida y aprender a construir nuevas maneras de interacción consigo mismos y con su entorno (15) (16).

Por otro lado, se sabe que el enfrentar cambios en el cuerpo genera sentimientos de ansiedad, angustia y depresión, debido a que los pacientes experimentan temor para relacionarse con otras personas de manera espontánea y libre. Según dos estudios realizados por Duque, et al., y Michelato, et al., existe evidencia que demuestra que factores como buenas relaciones sociales, ausencia de problemas psicológicos, actividades de ocio y ausencia de otros problemas relacionados a la salud ayudan a mejorar la calidad de vida, logrando un mejor enfrentamiento al nuevo entorno tanto físico como psicosocial (15) (16).

Así mismo, se debe orientar al paciente de manera integral hacia su nueva realidad o estilo de vida por medio del abordaje cognitivo especializado e interdisciplinario, incluyendo la ayuda por parte de enfermería, educación por parte del médico y la vivencia del proceso por experiencia de otros pacientes; todo esto con el fin de fortalecer las necesidades de la persona y promover el afrontamiento de la situación de una manera más eficaz por medio de la recuperación emocional, física y social (15) (16).

Es de suma importancia preparar al individuo desde antes de la realización de la cirugía, ya que presentará sentimientos de angustia y desesperanza a lo desconocido, por lo que el apoyo psicológico es vital, para así trabajar áreas que le ayuden a elaborar distintas destrezas frente a la enfermedad y conllevarla por medio de orientación constante.

Por último, en el postoperatorio es indispensable continuar con ayuda especializada en la cual también se le brinde educación y cuidados del estoma, con los cuales el paciente será capaz de afrontar de manera competente la identificación de complicaciones y problemas para poder llegar a la solución adecuada (15) (16).

\section{CONCLUSIONES:}

La realización de un estoma es un procedimiento que puede originar diversas complicaciones importantes para tomar en consideración en todo momento y así poder brindar un adecuado manejo.

Entre las consecuencias post quirúrgicas de un estoma se encuentra la transformación de una ostomía con débito normal en una de alto gasto. Se sabe que pacientes con esta condición tienen un incremento en la morbimortalidad y readmisión hospitalaria, lo cual se traduce en mayor costo para los sistemas de salud, ya que se requiere un manejo estricto en donde participen profesionales en medicina, nutrición, enfermería y psicología. 


\section{CIENCIA\&SALUD}

Actualmente se cuenta con muy pocos estudios sobre esta condición, por lo que se considera necesario realizar mayor investigación en cuanto al abordaje de la misma.

\section{REFERENCIAS BIBLIOGRÁFICAS:}

1. Merizalde, C. Larco, B. Cuidados del paciente con ostomía en centros geriatricos. Campaña "yo te integro". Proyecto integrador. 2019.

2. McGee M. Estomas. JAMA Network. 2016 mayo; 315(18).

3. Murken, D. Bleier, J. Ostomy-related complications. Clinics in colon and rectal surgery. 2019; 32(3).

4. Arenas J. López, C. Abilés, J. Rivera, R. Gándara, N. Utrilla, P. Protocol for the detection and nutritional management of high-output stomas. Nutrition Journal. 2015; 14(45).

5. Lee, Y. Kweon, M. Park, M. Nutritional management of a patient with a high-output stoma after extensive small bowel resection to treat Crohn's disease. Clinical nutrition research. 2019 julio; 8(3).

6. Goodey A. Colman, S. Safe management of ileostomates with high-output stomas. British Journal of Noursing. 2016 Agosto; 25(22).

7. Park J. Gessler, B. Block, M. Angenete, E. Complications and morbidity associated with loop ileostomies in patients with ulcerative colitis. Scandinavian Journal of Surgery. 2017 mayo; 107(1).

8. Kristensen K. Qvist, N. The actute effect of loperamide on ileostomy output: A randomized, double-blinded, placebo-control, crossover study. Basic and Clinical Pharmacology and Toxicology. 2017 junio; $121(6)$.

9. Takeda M. Takahashi, H. Haraguchi, N. Miyoshi, N. Hata, T. Yamamoto, H. et al. Factors predictive of high-output ileostomy: A retrospective single-center comparative study. Surgery Today. 2018 diciembre; 49.

10. Gaertner, W. Madoff, R. Mellgren, A. Kwaan, M. Melton, G. Postoperative diarrhea and high ostomy output impact postoperative outcomes after elective colon and rectal operations regardless of Clostridium difficile infection. The American Journal of Surgery. 2018 diciembre; 210(4).

11. Liu M. Tang, H. Yang, H. Chang, S. Is jejunostomy output nutrient or waste in short bowel syndrome? Experience from six cases. Asia Pacific Journal of Clinical Nutrition. 2016; 25(2).

12. De Vries F. Reeskamp, L. Van Ruler, O. Van Arum, I. Kuin, W. Dijksta, G. Systematic review: Pharmacotherapy for high-output enterostomies or enteral fistulas. Wiley. Alimentary, Pharmacology and Therapeutics. 2017 Abril; 46(3).

13. Rowe, K. Schiller, L. Ileostomy diarrhea: Pathophysiology and management. Taylor and Francis Group. 2020; 33(2).

14. Nagar A. Mehrotra, S. Yadav, A. Mangla, V. Lalwani, S. Mehta, N. Nundy, S. Distal bowel re-feeding in patients with proximal jejunostomy. The Society for Surgery of The Alimentary Tract. 2018 Mayo; 22(7).

15. Duque, P. Campiño, S. Vivencias de las personas portadoras de ostomía digestiva. Ciencia y Enfermería. Revista Iberoamericana de Investigación. 2019 Octubre; 25(10).

16. Michelato N. Dos Santos, M. Rodrigues, S. Galvao, C. Megumi, H. Aspectos psicológicos de pacientes ostomizados intestinales: Revisión integrativa. Revista Latinoamericana de Enfermagem. 2017; 25. 


\section{CIENCIA\&SALUD}

17. Arenas, J. Abilés, J. Utrilla, P. El alto débito por las ostomía: implicaciones clínicas, detección y manejo. 2015.

18. Vademecum. Vademecum (Online).; 2018 (citado 202012 20). Disponible en: https://www.google. co.cr/amp/s/www.vademecum.es/amp/principios-activos-loperamida-aO7daO3

19. De la Coba, C. Arguelles-Arias, F. De Argila, C. Júdez, J. Linares, A. Ortega-Alonso, A. et al. Efectos adversos de los inhibidores de la bomba de protones: revisión de evidencias y posicionamiento de la Sociedad Española de Patología Digestiva. 2016 Abril; 108 (4).

20. Chaves, N. Nivia, D. Uso de inhibidores de la bomba de protones y antagonistas de los receptores $\mathrm{H} 2$ de histamina como profilaxis en úlceras por estrés: una práctica justificada? Revista Colombiana de Enfermería. 2015 Octubre; 11

21. Zarate, A. Medina, S. Escuela de Medicina Universidad Finis Terrae (Online).; (citado 202012 20). Disponible en: https://mefinis.cl/img/manuales/ostomias.pdf

22. Arenas, J. Abilés, J. Moreno, G. Goitia, B. Navarro, P. Adán, N. Ostomías de alto débito: detección y abordaje. Nutrición Hospitalaria. 2014 Diciembre; 30 (6).

23. Victus. Cencomex (Online).; 2018 (citado 202012 22). Disponible en: https://www.cencomex.cl/ wp-content/uploads/2018/02/FICHA-TÉCNICA-GLUTAPAK-R-75555.pdf

24. Rivet, E. Ostomy Management A Model of Interdisciplinary Care. Science Direct. 2019 Octubre; 99 (5). 